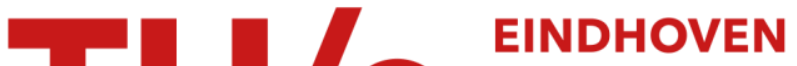 UNIVERSITY OF TECHNOLOGY
}

\section{Modal transmission-line modeling of propagation of plane radiowaves through multilater periodic building structures}

\section{Citation for published version (APA):}

Savov, S. V., \& Herben, M. H. A. J. (2003). Modal transmission-line modeling of propagation of plane radiowaves through multilater periodic building structures. IEEE Transactions on Antennas and Propagation, 51(9), 2244-2251. https://doi.org/10.1109/TAP.2003.817471

DOI:

10.1109/TAP.2003.817471

Document status and date:

Published: 01/01/2003

\section{Document Version:}

Publisher's PDF, also known as Version of Record (includes final page, issue and volume numbers)

\section{Please check the document version of this publication:}

- A submitted manuscript is the version of the article upon submission and before peer-review. There can be important differences between the submitted version and the official published version of record. People interested in the research are advised to contact the author for the final version of the publication, or visit the $\mathrm{DOI}$ to the publisher's website.

- The final author version and the galley proof are versions of the publication after peer review.

- The final published version features the final layout of the paper including the volume, issue and page numbers.

Link to publication

\section{General rights}

Copyright and moral rights for the publications made accessible in the public portal are retained by the authors and/or other copyright owners and it is a condition of accessing publications that users recognise and abide by the legal requirements associated with these rights.

- Users may download and print one copy of any publication from the public portal for the purpose of private study or research.

- You may not further distribute the material or use it for any profit-making activity or commercial gain

- You may freely distribute the URL identifying the publication in the public portal.

If the publication is distributed under the terms of Article 25fa of the Dutch Copyright Act, indicated by the "Taverne" license above, please follow below link for the End User Agreement:

www.tue.nl/taverne

Take down policy

If you believe that this document breaches copyright please contact us at:

openaccess@tue.nl

providing details and we will investigate your claim. 


\title{
Modal Transmission-Line Modeling of Propagation of Plane Radiowaves Through Multilayer Periodic Building Structures
}

\author{
Sava V. Savov, Senior Member, IEEE, and Matti H. A. J. Herben, Senior Member, IEEE
}

\begin{abstract}
The modal transmission-line (MTL) method is used to analyze the shielding effectiveness of buildings, which is an important parameter for the accurate planning of microcellular communication networks. For that, the buildings are modeled as multilayer lossy periodic structures. After a description of the extended MTL-theory, the newly developed MTL model is applied to brick walls, reinforced-concrete walls and concrete block walls. From a comparison of the MTL-results with previously published theoretical or experimental results it is concluded that the MTL method is very well suited, because it is accurate as well as computational time efficient
\end{abstract}

Index Terms-Full-wave methods, mobile communications, periodic structures, radiowave propagation, transmission-line modeling.

\section{INTRODUCTION}

$\mathbf{T}$ HE exponential growth of the number of users of mobile cellular systems states new problems to the planning of the network. A lot of research is done in this area to find a way for a more efficient use of the limited number of channels available within the cellular system. To increase the capacity of the mobile network, microcells are used in those areas where the traffic intensity is very high.

For an accurate planning of cellular networks, new radiowave propagation models for the estimation of the losses on the radio path must be used. For the conventional macrocells usually the position of the base station (BS) is higher than the average height of the surrounding buildings, but for microcells this height is very often below the rooftops of the buildings. In the microcellular concept, the shielding properties of the surrounding buildings are used to confine the radiated power within a small coverage area. While in the case of macrocells there are simple empirical models for the estimation of the path losses, for microcells the problem becomes very sitespecific. Therefore, more precise deterministic models must be used to represent better the physics of the propagation of the electromagnetic waves between the BS and the mobile station (MS).

There are several different mechanisms of propagation between BS and MS, such as line-of-sight propagation, reflection,

Manuscript received October 15, 2001; revised July 11, 2002. This work was supported in part by the Organization for Scientific Research in the Netherlands (NWO) and in part by the Dutch Ministry of Economic Affairs within the framework of the BraBant BreedBand (B4) project.

The authors are with the Faculty of Electrical Engineering, Eindhoven University of Technology, 5600 MB Eindhoven, The Netherlands (e-mail: S.V.Savov@tue.nl).

Digital Object Identifier 10.1109/TAP.2003.817471 and diffraction. These three mechanisms are included in most ray-tracing based field strength prediction tools [1], [2]. However at UHF frequencies, transmission of radiowaves through buildings surrounding the BS can be the dominant propagation mechanism [3]-[5]. Neglecting the contributions of these waves in the planning of a network may result in an unacceptable degree of inter-cell interference. There are very few publications available on the shielding effectiveness of buildings and they are based on rather simplified models [6]. To get a better insight in the way radiowaves propagate through buildings, a more accurate model is needed.

In the present paper this shielding effectiveness is studied in more detail, using one special property typical for many buildings: the periodicity. This property allows us to use a novel full-wave method, the so-called modal transmission-line (MTL) method, for an accurate and at the same time computationally efficient prediction of the shielding effect of walls and buildings [7].

This paper sets out with a description of the MTL method following the references [7]-[10]. Next, an extension of the theory is made by using matrix notations like in [11] and [12]. Then the extended theory is applied to the computation of the electromagnetic wave transmission through a simple single periodic layer, which can be a good model of some brick walls. Finally, the transmission properties of more complex walls are analyzed, namely reinforced-concrete walls, and concrete-block walls. In order to apply the MTL method to the latter types of walls, they are modeled as multilayer periodic structures. When available, the MTL results are compared with previously published computed or experimental results.

\section{MTL Method With a Plane WaVe Excitation}

In this section the MTL method will be introduced, following mainly the reference [8] for the case of a single-layer periodic structure and [11] for the case of a multilayer periodic structure. The geometry of the general scattering problem is shown in Fig. 1(a). While in [8] and [11] a characteristic equation for the propagation constants is used, here an eigenvalue equation is derived instead. The scattering problem is two-dimensional (2-D), with an infinite in $y$ direction periodic lossy dielectric structure. In this paper, only the TE case will be considered, but the method can also be applied to the TM case. A time-harmonic plane electromagnetic wave with an unit amplitude is incident on the periodic dichroic layer with a thickness $h$ at an angle of incidence $\theta$. A time convention for the harmonic field according to $\exp (-j \omega t)$ is used and suppressed. The rectangular profile 
shown is Fig. 1(a) is a very simple example of a periodic layer, but has many applications. The problem space consists of three regions (a, b, and c). In region (a) (air) $(l=0)$ there are two types of solutions namely incident and reflected waves, in region (c) (air) $(l=L+1)$ there are only transmitted waves, while in region (b) (periodic layers) $(l=1,2, \ldots, L)$ there are two kinds of solutions in the form of forward and backward traveling waves with respect to the $z$ direction. The structure has a periodicity in the $x$ direction with a period $d=d_{1 l}+d_{2 l}$, and the thickness of the whole structure is $h=\sum_{l=1}^{L} h_{l}$. Now it is convenient to use a global $x$ coordinate and a local $z_{l}$ coordinates for every layer, which means $x_{1}=x, z_{l}=z-\sum_{k=1}^{l-1} h_{k}(l=$ $0,1,2, \ldots, L) \quad\left(0 \leq z_{l} \leq h_{1}\right)$ (it is supposed that $\left.h_{0}=0\right)$. Then the solutions for the tangential fields $\left\{E_{y, l}, H_{x, l}\right\}$ in the $l$ th region can be written in terms of the Floquet space-harmonics with the following general form:

$$
\begin{aligned}
E_{l}\left(x, z_{l}\right)= & \sum_{n=-\infty}^{\infty}\left\{f_{l, n} e^{j k_{l, n} z_{l}}+b_{l, n} e^{j k_{l, n}\left(h_{l}-z_{l}\right)}\right\} \\
& \times \sum_{m=-\infty}^{\infty} a_{l, m n} e^{j k_{x, m} x}, \\
-H_{l}\left(x, z_{l}\right)= & \sum_{n=-\infty}^{\infty} y_{l, n}\left\{f_{l, n} e^{j k_{l, n} z_{l}}-b_{l, n} e^{j k_{l, n}\left(h_{l}-z_{l}\right)}\right\} \\
& \times \sum_{m=-\infty}^{\infty} a_{l, m n} e^{j k_{x, m} x}
\end{aligned}
$$

where $f_{l, n}$ are the amplitudes of the incident waves in the plane $z_{1}=0$, and $b_{l, n}$ are the amplitudes of the reflected waves in the plane $z_{l}=h_{l}$. Because there is a single plane wave it is logical that $f_{0, n}=\delta_{0 n}$ and $b_{0, n}=r_{n}$ with $\delta_{m n}$ the Kronecker-delta symbols $\left\{\delta_{m n}=1, m=n ; \delta_{m n}=0, m \neq n\right\}$ and $r_{n}$ the modal reflection coefficients, while for $l=L+1$ there are no reflected waves which means that $b_{L+1, n}=0$ and $f_{L+1, n}=t_{n}$ with $t_{n}$ the modal transmission coefficients. The longitudinal wavenumbers $\left\{k_{x, m}\right\}$, the transverse wavenumbers (modal propagation constants) $\left\{k_{l, n}\right\}$ and the modal characteristic admittances $\left\{y_{l, n}\right\}$ in the case of a homogeneous layer with a dielectric constant $\left\{\varepsilon_{r l} \equiv \varepsilon_{l}\right\}$ are (2) and (3), shown at the bottom of the page, where $k_{0}=\omega \sqrt{\varepsilon_{0} \mu_{0}}$ is the freespace wavenumber, $\eta_{0}=\sqrt{\mu_{0} / \varepsilon_{0}} \approx 377 \Omega$ is the free-space impedance. It can easily be verified, that this representation satisfies the periodic boundary conditions. Later on it will be shown that the propagation constants $\left\{k_{l, n}\right\}$ in the case of a periodic layer can be defined as eigenvalues of a suitable square matrix, and the elements $\left\{a_{l, m n}\right\}$ form a matrix of the corresponding eigenvectors as columns $\left[A_{l}\right]_{m n}=\left[a_{n}\right]_{m}$.
Fig. 1(a) shows that in every layer the complex dielectric constant $\varepsilon_{l}(x)$ is supposed to be a periodic function with the same period $d$. This means that the dielectric constant can be presented in terms of the following complex Fourier series:

$$
\begin{aligned}
\varepsilon_{l}(x) & =\sum_{n=-\infty}^{\infty} g_{l, n} e^{j n(2 \pi / d) x} \text { with } \\
g_{l, n} & =\frac{1}{d} \int_{0}^{d} \varepsilon_{l}(x) e^{-j n(2 \pi / d) x} d x .
\end{aligned}
$$

In case of dichroic lossy dielectric layers $(M=2)$ the coefficients of the Fourier series are

$$
\begin{aligned}
& g_{l, 0} \equiv \varepsilon_{l}=\frac{d_{1 l} \varepsilon_{1 l}+d_{2 l} \varepsilon_{2 l}}{d}, \text { (average) } \\
& g_{l, n}=j \frac{\varepsilon_{2 l}-\varepsilon_{1 l}}{2 \pi \cdot n}\left[1-e^{-j n(2 \pi / d) d_{1 l}}\right], \quad(n \neq 0) .
\end{aligned}
$$

For other periodic profiles (multichroic layers with $M \geq 3$ the coefficients can also be derived from (4).

Next, for every layer a square matrix $\left[P_{l}\right]$ is defined with elements

$$
P_{l, m n}=k_{0}^{2} g_{l, m-n}-k_{x, m a}^{2} \delta_{m n} .
$$

Starting with Helmholtz wave equation, the following eigenvalue equation is derived

$$
\operatorname{det}\left(\left[P_{l}\right]-k_{l, n}^{2}[I]\right)=0
$$

where $[I]$ is an identity matrix. It is obvious that in order to solve (7) numerically, the infinite matrices should be truncated so that $\left\{-N_{1} \leq n \leq N_{2}\right\}$. This means that instead of an infinite number of modes (plane waves) a finite number of $N$ modes is used in (1), where $N=N_{1}+N_{2}+1$. The number $N$ depends on the convergence properties of the series representation (1), but for the TE modes it is a relatively small number, as will be demonstrated in Section III of this paper by numerical experiments. An appropriate normalization of the elements of the matrix $\left[A_{l}\right]$ is performed to make the total power of all the modes equal to 1 . The unknown coefficients $\left\{f_{l, n} ; b_{l, n}\right\}$ in the series representation (1) can be determined by satisfying the boundary conditions for continuity of the tangential fields. Note that if the layer is homogeneous [(5) with $\varepsilon_{1 l}=\varepsilon_{2 l}=\varepsilon_{l}$ ] all the coefficients in the Fourier series are zero except $g_{l, 0}=$ $\varepsilon_{l}$. In this specific case the single-valued matrix $\left[P_{l}\right]$ simply reduces to $k_{l, n}^{2}$ as given by (2), and the matrix $\left[A_{l}\right]$ reduces to $[I]$. The modal propagation constants $\left\{k_{l, n}\right\}$ form a diagonal matrix $\left[K_{l}\right]$ with elements $\left[K_{l}\right]_{m n}=k_{l, n} \delta_{m n}$, while the modal

$$
\begin{aligned}
& k_{x, m}=k_{0} \sin \theta+m \frac{2 \pi}{d}, \quad(m=0, \pm 1, \pm 2, \ldots) \\
& k_{l, m}= \begin{cases}\sqrt{\varepsilon_{l} k_{0}^{2}-k_{x, m}^{2}}, & \left|k_{x, m}\right|<\sqrt{\varepsilon_{l}} k_{0}, \text { (propagating) } \\
j \sqrt{k_{x, m}^{2}-\varepsilon_{l} k_{0}^{2}}, & \left|k_{x, m}\right|>\sqrt{\varepsilon_{l}} k_{0}, \text { (evanescent) }\end{cases} \\
& y_{l, m}=\frac{k_{l, m}}{\left(k_{0} \eta_{0}\right)}
\end{aligned}
$$




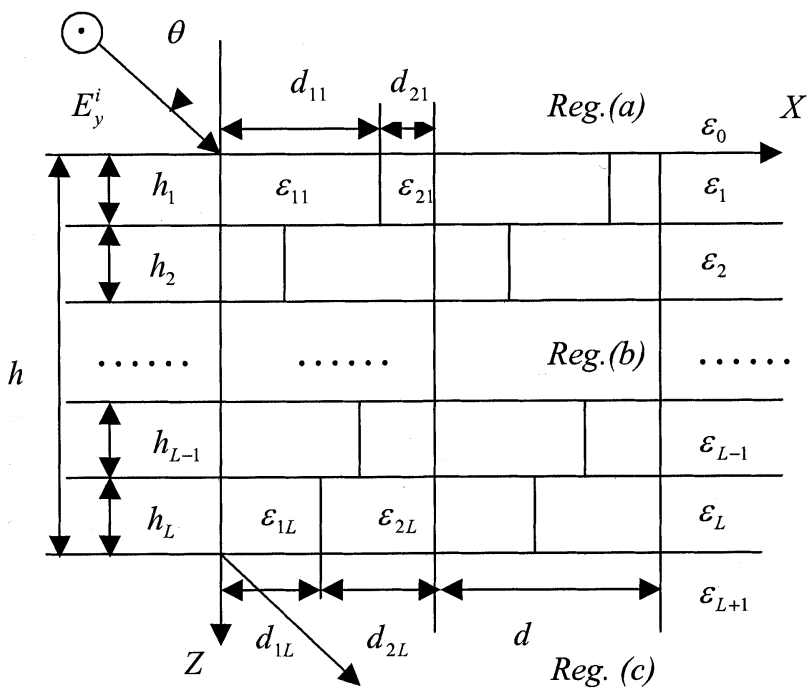

(a)

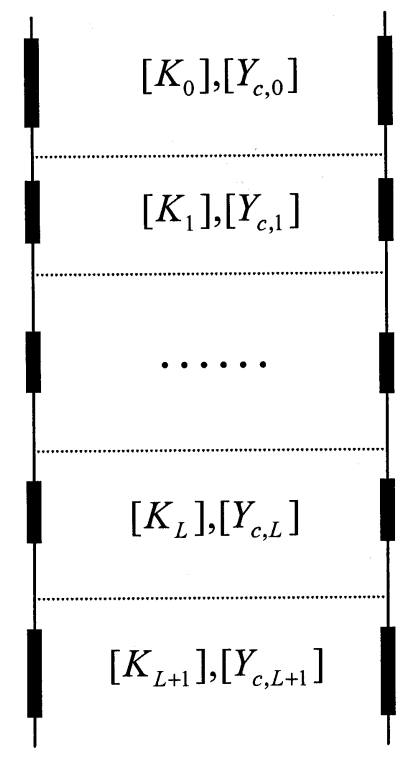

(b)

Fig. 1. (a) Geometry of the multilayer periodic structure and (b) equivalent MTL model.

characteristic admittances $\left\{y_{l, n}\right\}$ form another diagonal matrix $\left[Y_{c, l}\right]$ with elements $\left[Y_{c, l}\right]_{m n}=y_{l, n} \delta_{m n}$. They correspond to the equivalent transmission-line representation of the problem, shown in Fig. 1(b). The main difference with the classical transmission-line (TL) theory for multiple homogeneous layers is that here appropriate matrix characteristics $\left[K_{l}\right]$ and $\left[Y_{c, l}\right]$ are used instead of scalar ones.

The following finite-size local matrices in the $l$ th layer are introduced: a diagonal matrix of the layer propagator $\left[E_{l}\right]$ with elements $\left[E_{l}\right]_{m n}=\exp \left(j k_{l, n} h_{l}\right) \delta_{m n}$; square matrices of the transmission $\left[T_{l}\right]$, the reflection $\left[R_{l}\left(z_{l}\right)\right]$, and the admittance $\left[Y_{l}\left(z_{l}\right)\right]$. After performing lengthy, but straightforward matrix manipulations, based on imposing the boundary conditions for the continuity of the tangential components of the electromagnetic field, the following five steps matrix algorithm is derived for the $l$ th layer.
1) The admittance matrix (at $z_{l}=h_{l}$ ) is

$$
\left[Y_{l}\left(h_{l}\right)\right]=\left[Y_{l+1}(0)\right] .
$$

2) The reflection matrix (at $z_{l}=h_{l}$ ) is

$$
\begin{aligned}
{\left[R_{l}\left(h_{l}\right)\right]=\left\{\left[Y_{c, l}\right]+\right.} & {\left.\left[A_{l}\right]^{-1}\left[Y_{l}\left(h_{l}\right)\right]\left[A_{l}\right]\right\}^{-1} } \\
& \times\left\{\left[Y_{c, l}\right]-\left[A_{l}\right]^{-1}\left[Y_{l}\left(h_{l}\right)\right]\left[A_{l}\right]\right\} .
\end{aligned}
$$

3) The reflection matrix (at $z_{l}=0$ ) is

$$
\left[R_{l}(0)\right]=\left[E_{l}\right]\left[R_{l}\left(h_{l}\right)\right]\left[E_{l}\right] .
$$

4) The admittance matrix (at $z_{l}=0$ ) is

$$
\left[Y_{l}(0)\right]=\left[A_{l}\right]\left[Y_{c, l}\right]\left\{[I]-\left[R_{l}(0)\right]\right\}\left\{[I]+\left[R_{l}(0)\right]\right\}^{-1}\left[A_{l}\right]^{-1} \text {. }
$$

5) The transmission matrix of the layer is

$$
\left[T_{l}\right]=\left[A_{l}\right]\left\{[I]+\left[R_{l}\left(h_{l}\right)\right]\right\}\left[E_{l}\right]\left\{[I]+\left[R_{l}(0)\right]^{-1}\right\}\left[A_{l}\right]^{-1} .
$$

This procedure is applied in a backward recurrence, starting from the semi-infinite layer $(l=L+1)$ and ending to the semi-infinite layer $(l=0)$. For the latter one the transmission matrix is given by $\left[T_{0}\right]=[I]+[R]$.

Next, the total reflection $[R]$ and transmission matrix are introduced as

$$
[R]=\left[R_{0}(0)\right], \quad[T]=\left[T_{L}\right]\left[T_{L-1}\right] \ldots\left[T_{1}\right]\left[T_{0}\right] .
$$

The column matrices of the field transmission and reflection coefficients are then obtained by

$$
[t]=[T][u], \quad[r]=[R][u]
$$

where $[u]$ is a column matrix of the single-mode excitation $\left\{u_{n}=\delta_{n 0}\right\}$. Note that the algorithm gives the opportunity to find the field in every region by using (1).

The total power transmission and power reflection coefficients are by definition

$$
p_{t}=\sum_{n}\left|t_{n}\right|^{2}\left|\frac{y_{L+1, n}}{y_{L+1,0}}\right|, \quad p_{r}=\sum_{n}\left|r_{n}\right|^{2}\left|\frac{y_{0, n}}{y_{0,0}}\right| \text {. }
$$

The total power absorption coefficient is by definition

$$
p_{a}=1-p_{t}-p_{r}
$$

which in the case of lossless layers (real dielectric constants) is zero.

\section{Comparison Between the MTL Model AND THE TL MODEL PREDICTIONS}

The investigation will now be continued with the computation of the transmission properties of different kinds of walls. The MTL model must be used instead of the TL model because real walls are not homogeneous structures, but they are very often periodic structures: either single- or multilayer. First the method is applied to the simplest case of a single periodic layer, which can be a good approximation of some brick walls. In Section IV reinforced-concrete walls and concrete-block walls are analyzed, which can be classified as multilayer periodic structures. 
Consider plane wave transmission through a single periodic layer (Fig. 1(a) with $L=1$ ). For this case the convergence properties of the MTL-series representations (1) will be explored for the fields in the three regions. While in the case of a homogeneous wall three parameters appeared in the TL model (angle of incidence $\theta$, normalized thickness $h / \lambda$, dielectric constant $\varepsilon_{r}$ ) here, in the MTL model, three more parameters are added: normalized period $d / \lambda$, dichroic ratio $d_{1 l} / d$ and contrast $\left|\varepsilon_{2 l}-\varepsilon_{1 l}\right|$. The approximation of a homogeneous layer can be reached by approaching $h / d \rightarrow 0$, but it is also an average dielectric constant of the layer $\varepsilon_{l}(n=0)$ in the series (4), that reduces the MTL method to the conventional TL method. It is easy to see from (5), that these terms vanish also in two limiting cases for the dichroic ratio: 1) $d_{1 l} / d \rightarrow 0$ and 2) $d_{1 l} / d \rightarrow 1$, when the periodic layer becomes also a homogeneous one. This means that the most critical case for the convergence probably occurs for a ratio of about $1 / 2$, i.e., $d_{1 l}=d_{2 l}$. All these parameters influence the eigenvalues $\left\{k_{l, n}\right\}$ in (7), but also the elements of eigenvector matrix $\left[A_{l}\right]$ in (1). It should be noted that the propagator matrix $\left[E_{l}\right]$ also strongly depends on the thickness $h_{l}$ of the layer: a slower convergence is expected for larger values of the thickness. Further it is expected that the convergence will become slower with an increasing value of the period and the contrast.

The principal difference between the TL model and MTL model is that in case of a homogeneous layer, according to the TL model, the transmitted wave in reg. $(c)$ propagates in the same direction as the incident wave (the angle of incidence with the normal vector of the surface is $\theta$ ), while in case of a periodic layer the MTL model delivers a number of waves, all with their own direction of propagation $\varphi_{n}$, which can be determined from (2) to be $\varphi_{n}=\sin ^{-1}\left(k_{x, n} / k_{0}\right)=\sin ^{-1}(\sin \theta+n \lambda / d)$, $(n=0, \pm 1, \pm 2, \ldots)$. It is evident, that for some values of the modal index $n$, the angles are real (propagating modes), for other values the angles are complex (evanescent modes). It is obvious, that the scattering angle of the fundamental mode $(n=0)$ equals the angle of incidence (i.e., $\varphi_{0} \equiv \theta$ ), regardless the value of the normalized period $d / \lambda$. However, the width of the modal scattering pattern strongly depends on this parameter: in the limiting case $d / \lambda \rightarrow \infty$ (homogeneous layer) this width reduces to a single fundamental mode $(n=0)$, while in the case of small values of $d / \lambda$, the scattering pattern becomes wider. The normalized thickness $h / \lambda$ strongly influences the scattering amplitudes and consequently the power transmission coefficients of the modes $p_{t, n}$ [the components of the series (11)].

There are $N_{1}$ real angles related to $n<0$ and $N_{2}$ angles related to $n>0$. If $\theta=0, N_{1}=N_{2}$; if $\theta>0, N_{1}>N_{2}$; while in case $\theta<0, N_{1}<N_{2}$.

From this analysis the following estimation of the total number of modes $\left(N=N_{1}+N_{2}+1\right)$ can be obtained

$$
N \approx\left[\frac{2 d}{\lambda}\right]-1
$$

where the symbol $[x]$ means the largest integer, less than the real value $x$. This explains why the number $N$ is nearly linear proportional to the normalized period. Of course, this is an estimation of the number of propagating modes only; for more accurate calculations some evanescent modes have to

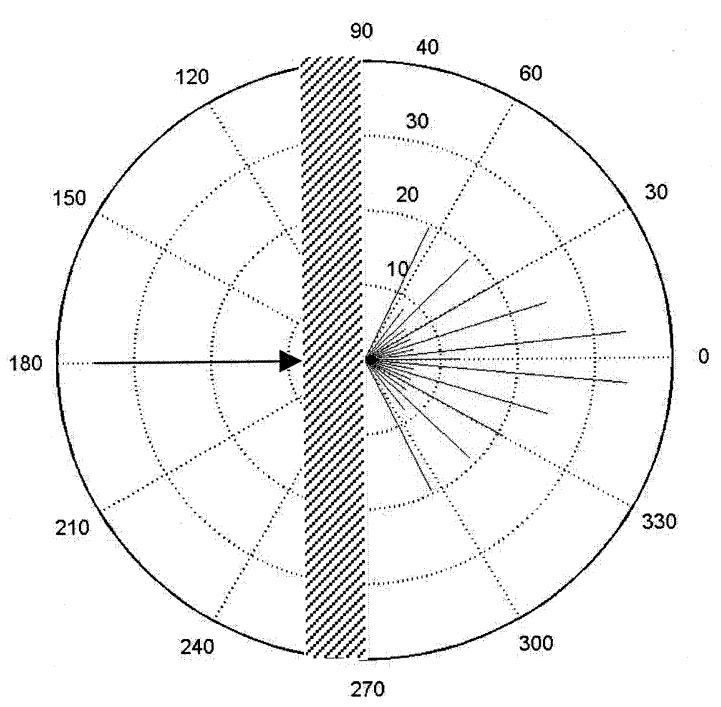

(a)

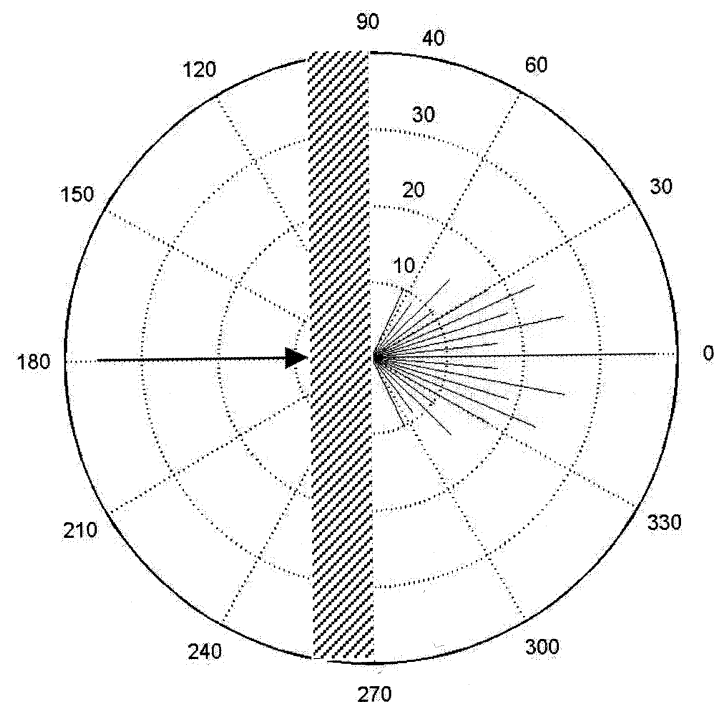

(b)

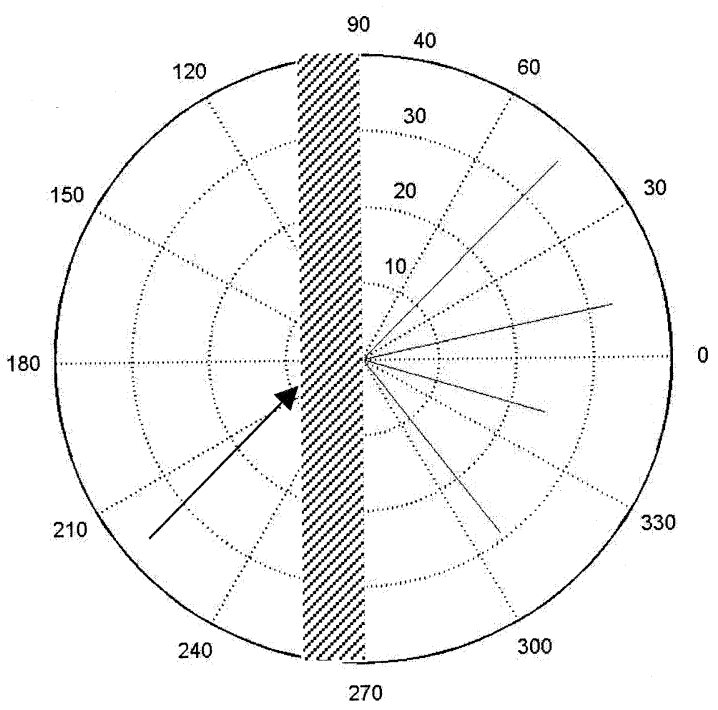

(c)

Fig. 2. Modal scattering patterns $(d B+40)$ of the transmitted waves through a single periodic layer with $\varepsilon_{l l}=5, \varepsilon_{21}=3, d_{11}=d_{21}(f=1.9 \mathrm{GHz})$ : (a) $\theta=0^{\circ}, d=10 \lambda, h=\lambda$. (b) $\theta=0^{\circ}, d=10 \lambda, h=10 \lambda$. (c) $\theta=45^{\circ}$, $d=2 \lambda, h=2 \lambda$. 


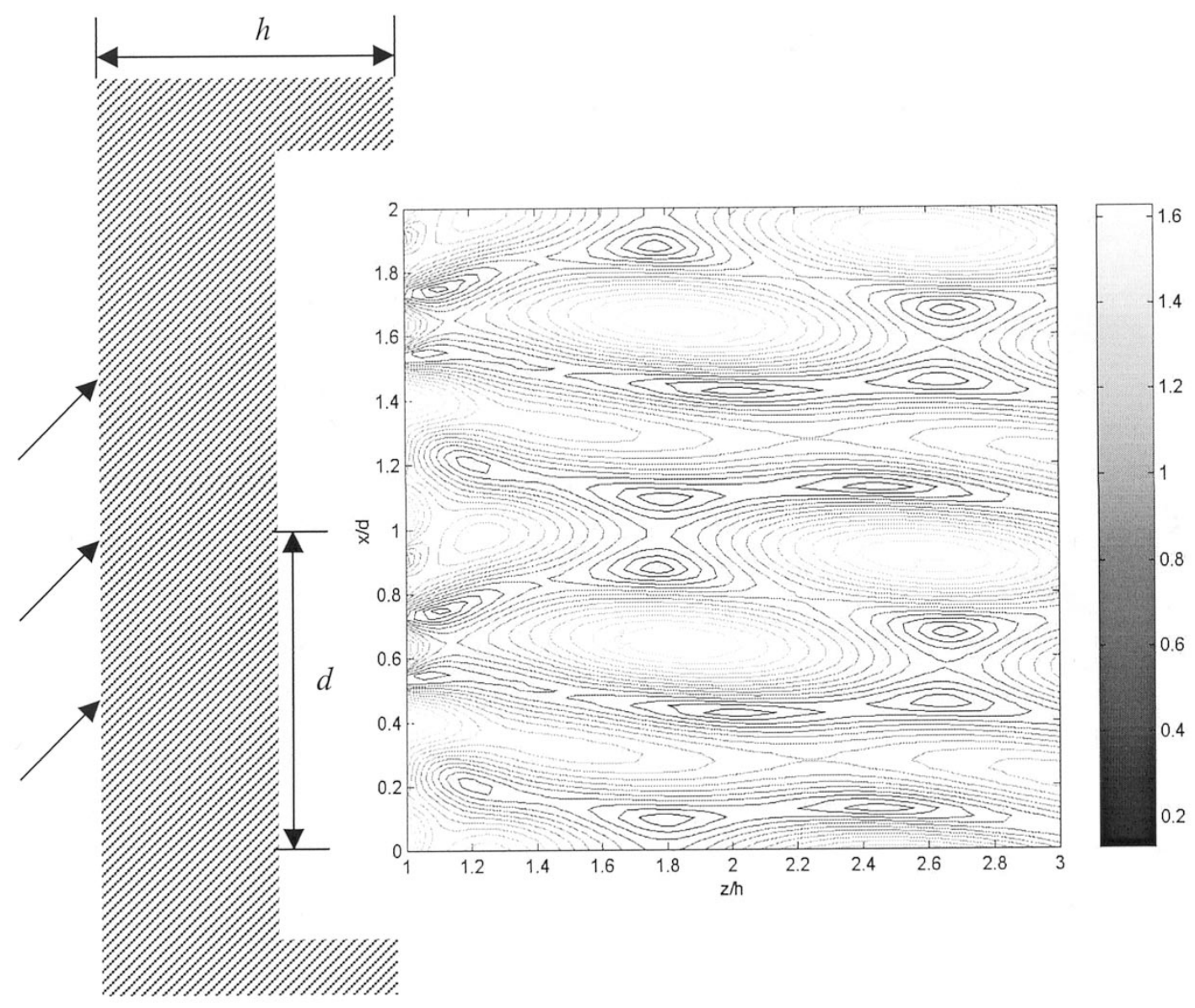

Fig. 3. Electric field distribution $(\mathrm{V} / \mathrm{m})$ behind the periodic layer of Fig. $2(\mathrm{c})$ versus normalized coordinates $x / d, z / h\left(E^{i}=1 \mathrm{~V} / \mathrm{m}\right)$.

be included, which mean that the estimation from (13) must be slightly enhanced. The scattering pattern of the modes in region $(c)$ is interesting also for getting a better physical insight of the transmission problem.

Therefore, Fig. 2 presents scattering patterns of a periodic dielectric slab with parameters: ratio $d_{11} / d=0.5$, dielectric constants $\varepsilon_{11}=5, \varepsilon_{21}=3$, at a frequency $f=1.9 \mathrm{GHz}$ (wavelength $\lambda \approx 15.8 \mathrm{~cm}$ ) obtained from simulations based on the MTL model. The results shown in Fig. 2(a) hold for the case of a period $d=10 \lambda$, a thickness $h=\lambda$, and normal incidence $\left(\theta=0^{\circ}\right)$. The number of propagating modes $N=19(n=$ $0, \pm 1, \ldots, \pm 9)$ agrees very well with the estimation of (13). The pattern shows that the fundamental mode is not dominant here, but the modes with index \pm 1 . To explore the effect of a larger thickness, Fig. 2(b) shows the scattering pattern for a layer with thickness $h=10 \lambda$, again for normal incidence. It is clearly visible that the number of propagating modes and their angles remain the same, but the scattering amplitudes are different: now the fundamental mode is the dominant one. Finally, Fig. 2(c) shows the results for the case of thickness and period compared to the wavelength $h=d=2 \lambda$ and oblique wave incidence with $\theta=45^{\circ}$. Now, the number of propagating modes $N=4$ [also close to the estimation made by (13)] and the maximum power is transmitted in the direction of the fundamental mode $\varphi_{0}=45^{\circ}$. Fig. 3 shows the corresponding field distribution behind the layer, which clearly shows the periodic interference pattern of the transmitted plane waves.

\section{APPLiCATIONS}

\section{A. Reinforced-Concrete Walls}

These walls are made up of a steel wire-grid embedded in a thick slab of concrete. It is particularly important to compare the electromagnetic performance of concrete walls and reinforced-concrete walls and to evaluate the influence of the steel grid. In [13] the finite element method (FEM) is used for analyzing such structures, which makes the analysis more complicated and time-consuming. Here the periodicity of the structure is exploited and the MTL method is applied.

The geometry of such wall is a particular case of the general geometry, shown in Fig. 1(a), where the wire-diameter is $2 a$. Because of the application of the MTL model it is necessary to replace the circular wire-grid with a square wire-grid with a size, equal to the wire-diameter. Then the reinforced-concrete structure can be modeled as a simple three-layer structure $(L=3)$. Computer simulations showed that this simplification is valid. In order to be able to compare the MTL-results with those obtained from FEM as published in [13], a structure with similar dimensions will be analyzed. This means that the thickness of the inhomogeneous layer $h_{2}=d_{12}=3 \mathrm{~mm}, d_{22}=d-d_{12}$, 


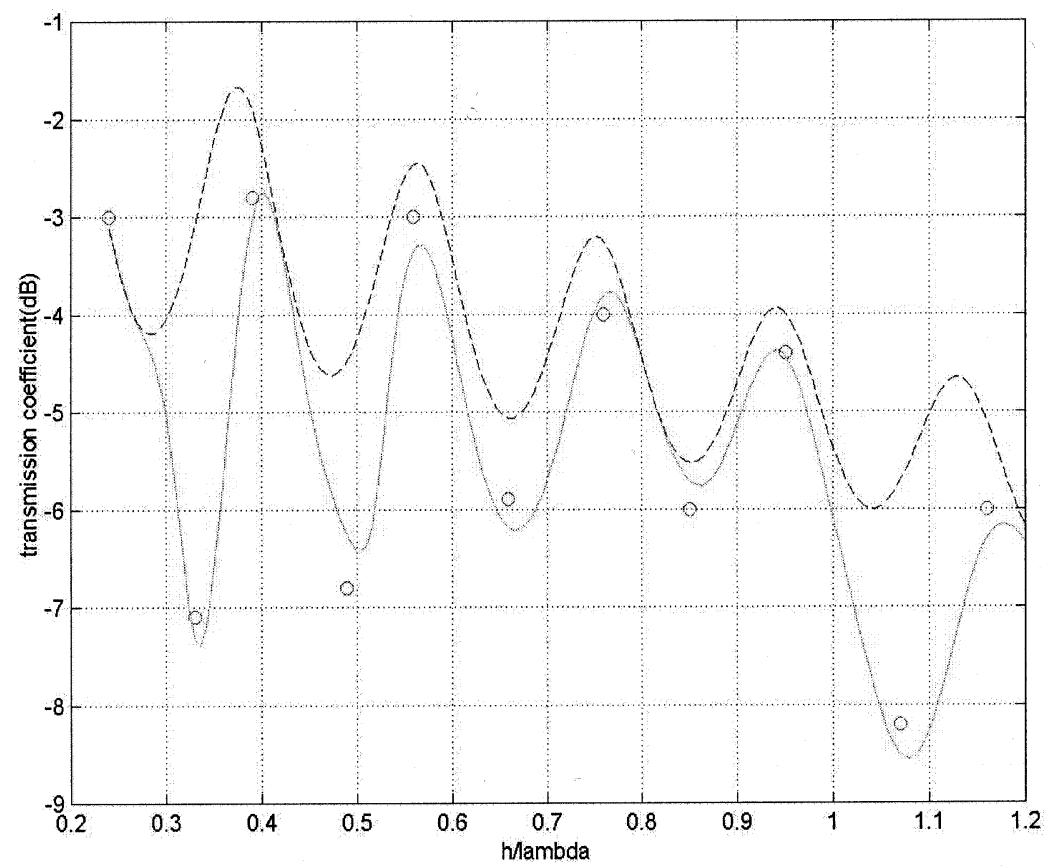

(a)

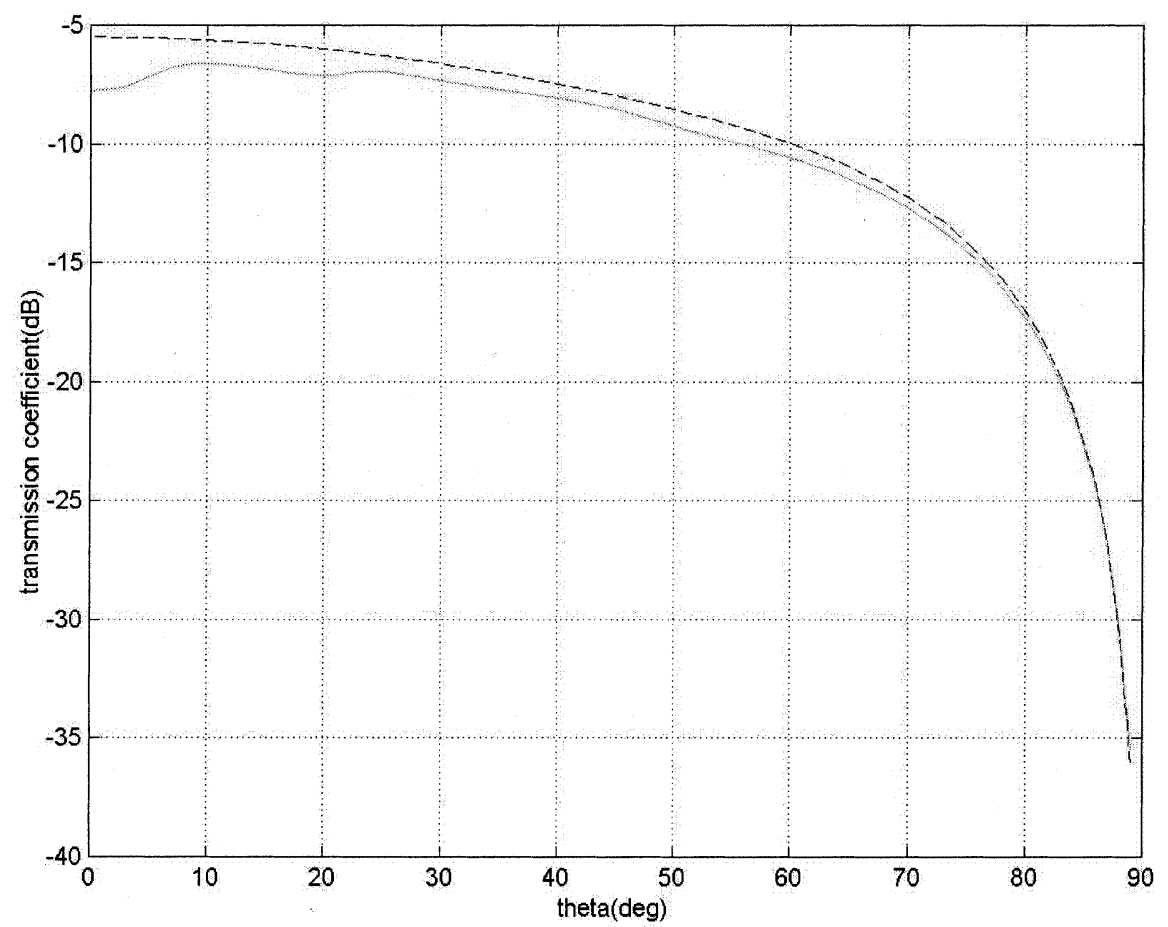

(b)

Fig. 4. (a) Transmission coefficient (dB) of a reinforced-concrete wall versus normalized thickness $h / \lambda$ for normal incidence $(f=1.8 \mathrm{GHz})$ : (००० FEM [13], ---- homogeneous wall). (b) Transmission coefficient (dB) of a reinforced-concrete wall versus angle of incidence $\theta$, thickness $h=1.8 \mathrm{~cm}(f=1.8 \mathrm{GHz})$ : $(----$ homogeneous wall).

where the period $d=15 \mathrm{~cm}$, while the thickness of other two homogeneous layers $h_{1}=h_{3}=\left(h-h_{2}\right) / 2$. The complex dielectric constant of the concrete $\varepsilon_{r}=7+j 0.3$ [14], which means $\varepsilon_{11}=\varepsilon_{12}=\varepsilon_{22}=\varepsilon_{31}=\varepsilon_{32}=\varepsilon_{r}$, the conductivity of the steel $\sigma=1.11 \times 10^{6} \mathrm{~S} / \mathrm{m}$ [15], [16] and the frequency of the incident wave $f=1.8 \mathrm{GHz}$ (wavelength $\lambda \approx 16.67 \mathrm{~cm}$ ). The complex dielectric constant of the metal sublayer $\varepsilon_{2 l}=$ $\hat{\varepsilon}_{r}=j \sigma\left(\omega \varepsilon_{0}\right) \approx j 1.11 \times 10^{7}$, i.e., the contrast is extremely large. Fig. 4(a) shows the transmission coefficient of both the reinforced-concrete wall (solid line) and the homogeneous concrete wall with the same thickness $h$ (broken line), as a function of normalized wall thickness $h / \lambda(4 \mathrm{~cm}<h<20 \mathrm{~cm})$, for normal wave incidence $\left(\theta=0^{\circ}\right)$. Here $N=51$ modes are needed, which is much more than the estimation $N=2$ from (13), because of the presence of a conductor, but even then the method remains efficient. The results for the reinforced-con- 


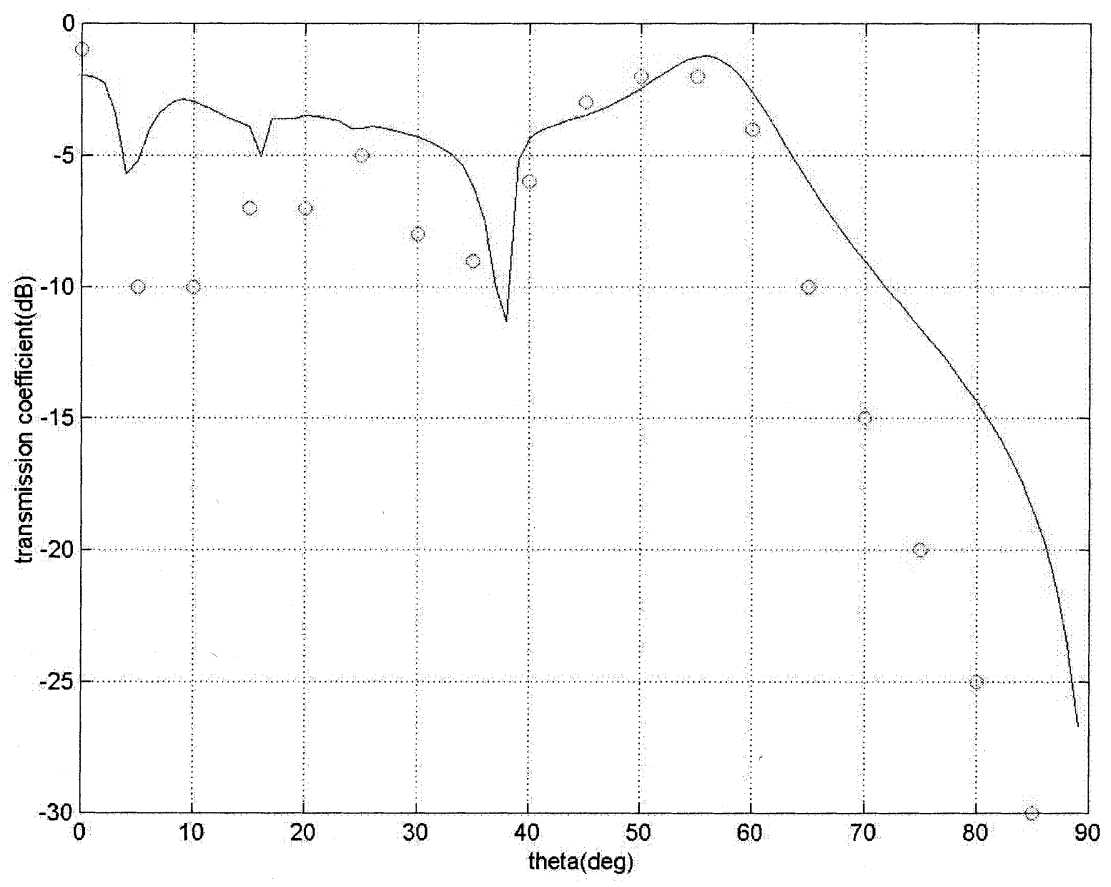

Fig. 5. Transmission coefficient (dB) of a concrete-block wall versus angle of incidence $\theta$, thickness $h=15 \mathrm{~cm}(f=3 \mathrm{GHz})$ : (o ० ० experiment [17]).

crete wall obtained by the MTL method are very close to those obtained by the FEM in [13, Fig. 12(b)] and added to Fig. 4(a) as circles. This clearly demonstrates the accuracy of the MTL method. It will also be clear from this figure that the wire-grid increases the shielding effectiveness of the wall (the path losses are increased), which is expected, but on average the increase is less than $2-3 \mathrm{~dB}$ for this particular value of $d_{12} / \lambda=0.018$. Another interesting observation is, that the transmission coefficient fluctuates when the wall thickness increases, which means that it is possible to receive more transmitted power in case the wall is thicker, because of the resonant behavior of the wall.

Fig. 4(b) presents the transmission coefficient as a function of the angle of incidence for the wall with and without wire-grid both having a thickness of $h=18 \mathrm{~cm}$ and for the same frequency as in Fig. 4(a). Here again with a solid line are presented the MTL- results, with a dashed line-the TL results. It can be concluded from this figure that as expected for both wall losses increase with increasing angle of incidence and that the losses are larger for the reinforced-concrete wall than those for the homogeneous concrete wall for all possible angles of incidence. However, the difference decreases with increasing angle of incidence because the effective normalized period $d \cdot \cos \theta / \lambda$ also decreases.

\section{B. Concrete-Block Walls}

The geometry of a concrete-block wall is a particular case of the general geometry, shown in Fig. 1(a) in the case of three layers $(L=3)$. In some analyses this type of walls is considered to be approximately homogeneous with average parameters. This so-called low-frequency approximation is only valid in case the wavelength is larger than the dimensions of the wall's ingredients [14], which is true in some extent at the frequency $f=0.9 \mathrm{GHz}(\lambda \approx 33.3 \mathrm{~cm})$, but seems not to be a very good approximation at the frequency $f=1.9 \mathrm{GHz}(\lambda \approx 15.8 \mathrm{~cm})$ and will not be true for even higher frequencies. The structure of the walls (which usually is periodic) becomes an important factor at these frequencies.

This problem is already treated in [17] for some specific cases by using the space harmonics. The same structure will now be analyzed with the MTL method. This means that the dimensions of the ingredients are: $h_{1}=h_{3}=1.35 \mathrm{~cm}$, $h_{2}=12.6 \mathrm{~cm}, \quad(h=15.3), d_{12}=11 \mathrm{~cm}, d_{22}=4 \mathrm{~cm}$ $(d=15 \mathrm{~cm})$ and the dielectric constant of the cinder block at a frequency $f=3 \mathrm{GHz}(\lambda=10 \mathrm{~cm})$ is taken [14] $\varepsilon=3+j 0.03$ (small losses are included). Here, $\varepsilon_{11}=\varepsilon_{12}=$ $\varepsilon_{22}=\varepsilon_{31}=\varepsilon_{32}=\varepsilon_{r}$, while $\varepsilon_{21}=1$ (air enclosure). Now the three-layer MTL model needs $N=3$ modes only, which is in good agreement with (13), and the angular response of the transmission coefficient (-shielding effectiveness) is shown in Fig. 5. It can be observed that the theoretical predictions (solid line) are well correlated with the experimental results (circles), taken from [17, Fig. 17]. The difference can be explained by the fact that during the experiments the incident wave was cylindrical, while in the MTL method a plane incident wave was supposed. Therefore, the agreement could be further improved by introducing a line-source in the MTL method, which in principle can be done by using the plane-wave expansion of a cylindrical wave. Furthermore, numerical experiments demonstrated that the transmission coefficient of this composite structure is rather sensitive to its complex dielectric constant, which is not known very accurately, because it depends on many factors.

\section{CONCLUSION}

The MTL method has been successfully applied to the analysis of the shielding effectiveness of buildings, which is an important parameter for the accurate planning of microcellular 
mobile networks. For that, a special property typical for many building structures is exploited namely the periodicity. In addition to the transmission losses, the novel MTL method also yields the spectral distribution of the transmitted radiowaves, from which the complete field distribution behind a building structure can be obtained. It is clear from the theoretical description of the MTL method in Chapter 2 of the paper that this method can also be used to determine the reflection properties of such walls. The accuracy of the MTL method was examined in Chapter 4 of the paper by comparing the MTL-results with previously published theoretical or experimental results for reinforced-concrete walls and concrete-block walls. This study justifies the conclusion that for the modeling of propagation of plane radiowaves through simple multilayer periodic building structures, the MTL method is well suited because it is accurate as well as computational time efficient. Additional measurements are needed to verify the method in the case of more complex inhomogeneous building structures.

\section{REFERENCES}

[1] G. A. J. van Dooren and M. H. A. J. Herben, "A deterministic approach for the modeling of wave propagation around buildings," J. Electromagn. Waves Appl., vol. 8, pp. 175-194, 1994.

[2] S. V. Savov, J. H. Whitteker, and R. Vasilev, "Attenuation of waves behind a building," Proc. Inst. Elect. Eng. Microw. Antennas Propagation, vol. 146, pp. 145-149, Apr. 1999.

[3] Y. L. C. de Jong, M. H. A. J. Herben, J. F. Wagen, and A. Mawira, "Transmission of UHF radiowaves through buildings in urban microcell environments," Elect. Lett., vol. 35, no. 9, pp. 743-745, 1999.

[4] Y. L. C. de Jong and M. H. A. J. Herben, "High-resolution angle-ofarrival measurement of the urban mobile radio channel," IEEE Trans. Antennas Propagat., vol. 47, pp. 1677-1687, Nov. 1999.

[5] Y. L. C. de Jong, M. H. J. L. Koelen, and M. H. A. J. Herben, "Measurement of building transmission loss using wide-band radio channe sounding," Electron. Lett., vol. 36, no. 11, pp. 1067-1069, June 2000.

[6] E. Damosso and L. Correira, Eds., "COST 231 Final Report," Commission of the European Community, Torino, Italy, 1996.

[7] S. V. Savov and M. H. A. J. Herben, "Modal transmission-line calculation of the shielding effectiveness of composite structures," Electron. Lett., vol. 37, no. 8, pp. 487-488, Apr. 2001.

[8] H. Bertoni, L. Cheo, and T. Tamir, "Frequency-selective reflection and transmission by a periodic dielectric layer," IEEE Trans. Antennas Propagat., vol. 37, pp. 78-83, Jan. 1989.

[9] S. Peng, T. Tamir, and H. Bertoni, "Theory of periodic dielectric waveguides," IEEE Trans. Microwave Theory Tech., vol. 23, pp. 123-133, Jan. 1975.

[10] L. Lewis and A. Hessel, "Propagation characteristics of periodic arrays of dielectric slabs," IEEE Trans. Microwave Theory Tech., vol. 19, pp. 276-286, Mar. 1971.
[11] T. Tamir and S. Zhang, "Modal transmission-line theory of multilayered grating structures," J. Lightwave Technnol., vol. 14, pp. 914-927, May 1996.

[12] L. Li, "Multilayer modal method for diffraction gratings of arbitrary profile,depth and permitivity," J. Opt. Soc. Amer. A, vol. 10, pp. 2581-2591, Dec. 1993.

[13] E. Richalot, M. Bonilla, W. Man-Fai, V. Fouad-Hanna, H. Baudrand, and J. Wiart, "Electromagnetic propagation into reinforced-concrete walls," IEEE Trans. Microwave Theory Tech., vol. 48, pp. 357-366, Mar. 2000.

[14] C. L. Holloway, P. L. Perini, R. R. DeLyser, and K. C. Allen, "Analysis of composite walls and their effects on short-path propagation modeling," IEEE Trans. Veh. Technol., vol. 46, pp. 730-738, Aug. 1997.

[15] A. R. von Hippel, Dielectric Materials and Applications. Cambridge, MA: M.I.T. Press.

[16] Y. Lo and S. Lee, Antenna Handbook. New York: Chapman and Hall, 1993.

[17] W. Honcharenko and H. L. Bertoni, "Transmission and reflection characteristics atconcrete block walls in the UHF bands proposed for future PCS," IEEE Trans. Antennas Propagat., vol. 42, pp. 232-239, Feb. 1994.

Sava V. Savov (M'91-SM'98) was born in Dobrich, Bulgaria, on January 11, 1951. He received the M.Sc. degrees both in electrical engineering and applied mathematics from Technical University of Sofia, Sofia, Bulgaria, in 1974 and 1976, respectively, and the Ph.D. degree in electrical engineering from Technical University of Varna, Varna, Bulgaria, in 1991.

In 1978, he joined the Institute of Electronics, Sofia, as a Research Assistant. In 1983, he was appointed as an Assistant Professor in the Department of Mathematics, Technical University of Varna, where, since 1989, he has been with the Department of Electronic Engineering and is currently an Associate Professor. His research interests are in the area of computational eleetromagnetics, numerical modeling of antennas and propagation in wireless communications.

Dr. Savov has been a Visiting Researcher at the Center for Personal Communications, Aalborg University, Denmark, at the Communication Research Center, Ottawa, Canada, and also at the Radiocommunications Group, Eindhoven University of Technology, The Netherlands. He took an active participation in the establishment of the Bulgarian Section of the IEEE and its first joint Bulgarian Chapter on ED/MTT/AP in 1995.

Matti H. A. J. Herben (S'80-M'83-SM'88) was born in Klundert, The Netherlands, in 1953. He received the M.Sc. degree (cum laude) in electrical engineering and the Ph.D. degree in technical sciences from Eindhoven University of Technology (EUT), The Netherlands, in 1978 and 1984, respectively.

Since 1978, he has been with the Radiocommunications Group of EUT, currently as an Associate Professor. His research interests and publications are in the areas of design and numerical analysis of reflector and lens antenna systems, radio interference reduction, electromagnetic wave propagation on terrestrial and satellite links, remote sensing of the turbulent troposphere, and microwave radiometry.

Dr. Herben is a Member of the Royal Institute of Engineers (KIvI), the Netherlands Electronics and Radio Society (NERG), and the Dutch URSI-Committee. He was an Associate Editor of Radio Science from 1993 through 1996. 